\title{
Numerical simulations of flux flow in stacked Josephson junctions
}

\author{
Madsen, Søren Peder; Pedersen, Niels Falsig
}

Published in:

Physical Review B Condensed Matter

Link to article, DOI:

10.1103/PhysRevB.72.134523

Publication date:

2005

Document Version

Publisher's PDF, also known as Version of record

Link back to DTU Orbit

Citation (APA):

Madsen, S. P., \& Pedersen, N. F. (2005). Numerical simulations of flux flow in stacked Josephson junctions. Physical Review B Condensed Matter, 72(13), 134523. https://doi.org/10.1103/PhysRevB.72.134523

\section{General rights}

Copyright and moral rights for the publications made accessible in the public portal are retained by the authors and/or other copyright owners and it is a condition of accessing publications that users recognise and abide by the legal requirements associated with these rights.

- Users may download and print one copy of any publication from the public portal for the purpose of private study or research.

- You may not further distribute the material or use it for any profit-making activity or commercial gain

- You may freely distribute the URL identifying the publication in the public portal

If you believe that this document breaches copyright please contact us providing details, and we will remove access to the work immediately and investigate your claim. 


\title{
Numerical simulations of flux flow in stacked Josephson junctions
}

\author{
S. Madsen* and N. F. Pedersen ${ }^{\dagger}$ \\ Oersted DTU, Section of Electric Power Engineering, Technical University of Denmark, DK-2800, Kgs. Lyngby, Denmark
}

(Received 21 June 2005; published 25 October 2005)

\begin{abstract}
We numerically investigate Josephson vortex flux flow states in stacked Josephson junctions, motivated by recent experiments trying to observe the vortices in a square vortex lattice when a magnetic field is applied to layered high- $T_{c}$ superconductors of the $\mathrm{Bi}_{2} \mathrm{Sr}_{2} \mathrm{CaCl}_{2} \mathrm{O}_{x}$ type. By extensive numerical simulations, we are able to clearly distinguish between triangular and square vortex lattices and to identify the parameters leading to an in-phase vortex configuration.
\end{abstract}

DOI: 10.1103/PhysRevB.72.134523

PACS number(s): 74.50. $+\mathrm{r}, 05.45 . \mathrm{Yv}, 85.25 . \mathrm{Cp}$

\section{INTRODUCTION}

The intrinsic Josephson effect in the layered high- $T_{c}$ superconductor $\mathrm{Bi}_{2} \mathrm{Sr}_{2} \mathrm{CaCu}_{2} \mathrm{O}_{x}(\mathrm{BSCCO})$ has been a subject of intense research in the past decade. ${ }^{1,2}$ These atomically layered superconductors of the so-called BSCCO family behave as stacks of Josephson junctions. The basic properties of a stack of Josephson junctions may be described by the inductive coupling model ${ }^{3}$ or the charge polarization model. ${ }^{4}$ The system has, even in zero magnetic field, very complicated dynamics as seen in the studies of coupled sine-Gordon equations. $^{3,5,6}$ When a magnetic field is applied along the layer direction, it may generate Josephson vortices in the junctions, resulting in a Josephson vortex lattice. Recently, the dynamic properties of this lattice in BSCCO have been the subject of extensive experimental research. ${ }^{7-9}$ Quite typically, magnetic field oscillations of the flux flow voltage or the flux flow resistance with either a $\Phi_{0}$ or a $\Phi_{0} / 2$ periodicity (in applied field times the layer area) has been observed. A straightforward explanation of such periodicities can be obtained by looking at the lattice structure. The vortex lattice is driven along the layers by the Lorentz force from an applied bias current. Since this vortex flow gives rise to a voltage, a periodic oscillation (in magnetic field) of the flux flow voltage can be expected. If fluxons in each layer leave simultaneously $\left(\Phi_{0}\right.$ periodicity), it is an indication of a square vortex lattice (in-phase vortex configuration). If, on the other hand, a $\Phi_{0} / 2$ period is observed, it is an indication of a triangular vortex lattice ${ }^{7}$ (antiphase vortex configuration). The crucial test for this explanation is of course a direct measurement of the frequency and power of the electromagnetic radiation emitted by the vortices leaving the sample.

Recently, attempts to fabricate a microwave oscillator based on the in-phase motion (square lattice) of vortices in single crystals of the $\mathrm{Bi}_{2} \mathrm{Sr}_{2} \mathrm{CaCu}_{2} \mathrm{O}_{x}$ type has been reported. ${ }^{9}$ The frequency range is typically in the terahertz range which makes the ideas potentially very interesting but, at the same time, experimentally difficult to verify. So far, no direct measurement of emitted $\mathrm{THz}$ radiation has been reported. Various schemes to enhance the radiation, even with a triangular vortex lattice, were reported in Refs. 10-12.

In a recent paper, Ref. 13, a numerical simulation was done for a single, long Josephson junction. Both periodicities, $\Phi_{0}$ and $\Phi_{0} / 2$, were observed, which is very similar to experiments and numerical simulations on stacks. Since the concepts of square and triangular vortex lattice have no meaning in a single junction, it was concluded that there is no direct connection between the periodicity and the vortex lattice ordering. This was also confirmed by numerical simulations on 5 and 10 layer stacks. ${ }^{11,12}$ In Ref. 13, the origin of both the $\Phi_{0}$ and the $\Phi_{0} / 2$ oscillations was identified as being due to Fiske modes, ${ }^{14}$ which are known to exist in BSCCO stacks. ${ }^{15,16}$

In this paper, we present numerical calculations trying to illuminate the conditions for the formation of a square vortex lattice in Josephson junction stacks. By looking directly at the phases of the individual junctions, we are able to unambiguously identify the lattice structure in the voltage vs applied magnetic field characteristics.

\section{THE MODEL}

The geometry of the system under consideration is shown in Fig. 1. With identical parameters for the individual junctions in the stack, the equations with $N+1$ superconducting layers and $N$ insulating layers may be written as $^{3}$

$$
\boldsymbol{J}=\boldsymbol{S}^{-1} \boldsymbol{\phi}_{x x}
$$

where the $i$ th element of $\boldsymbol{\phi}, \phi^{i}$, is the gauge invariant phase difference across insulating layer $i . S$ is a $N \times N$ matrix, expressing the inductive coupling between the junctions, thus

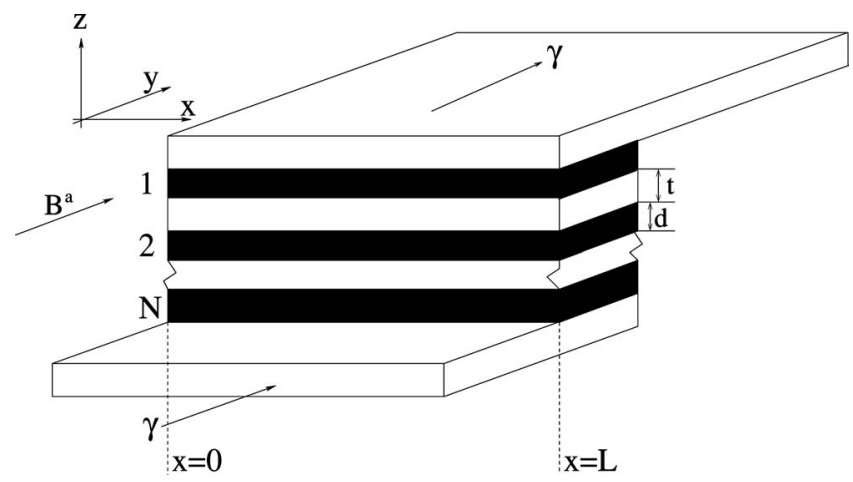

FIG. 1. The geometry of the stacked junctions. Black layers are insulators with thickness $d$ and white layers are superconductors with thickness $t$. The applied magnetic field $B^{a}$ is in the $y$ direction. 


$$
S=\left(\begin{array}{ccccc}
1 & S & & & \\
S & 1 & S & & \\
& S & 1 & S & \\
& & \ddots & \ddots & \ddots
\end{array}\right),
$$

with $S$ being the numerical value of the coupling, given by ${ }^{3}$

$$
S=-\frac{\lambda}{d \sinh (t / \lambda)+2 \lambda \cosh (t / \lambda)},
$$

where $\lambda$ is the London penetration depth. The components of $J$,

$$
J^{i}=\phi_{t t}^{i}+\alpha \phi_{t}^{i}+\sin \phi^{i}-\gamma,
$$

are expressing the current, in the $z$ direction, across insulating layer number $i$, where the $\alpha$ term is a dissipative term, and $\gamma$ is the applied bias current. The model does not describe in-plane dissipation, which may be important for direct comparison with experiments on BSCCO (see Refs. 17 and 18).

Equations (1)-(4) have been written in normalized units. Space $x$ is normalized to the Josephson penetration depth, $\lambda_{J}=\sqrt{\hbar / 2 e \mu_{0} J^{c} d^{\prime}}$, and time $t$ is normalized to the inverse plasma frequency, $\omega_{0}^{-1}=\sqrt{\hbar C / 2 e J^{c}}$, where the symbols have their usual meaning. See Ref. 19 for details.

Boundary conditions for Eqs. (1)-(4) in an applied magnetic field may be written as ${ }^{3}$

$$
\phi_{x}^{i}(0, t)=\phi_{x}^{i}(L, t)=(1+2 S) B^{a} \equiv \Gamma,
$$

where $i=1, \ldots, N$ and $B^{a}$ is the applied (dimensionless) magnetic field, assuming an ideal overlap geometry, neglecting the magnetic field generated by the bias current. We have assumed identical junctions, which might not always be the case in artificial stacks. When, for example, $N=2$, the junctions are usually having a very thin middle superconducting layer but thick outer superconducting layers. This changes the factor in front of $B^{a}$ to $(1+S)$, since the inductive coupling through the thick superconducting layers is zero. To avoid this ambiguity, we use the variable $\Gamma$ in our calculations.

\section{TWO STACKED JUNCTIONS}

The case of $N=2$ has previously been investigated analytically by Grønbech-Jensen et al. in Ref. 20. Using the adiabatic perturbation technique and trial functions of the forms

$$
\phi^{1}=\phi_{0}^{1}+\Gamma x+\omega t+\delta \phi^{1}
$$

and

$$
\phi^{2}=\phi_{0}^{2}+\Gamma x+\omega t+\delta \phi^{2},
$$

where $\delta \phi^{i}$ are small perturbations taken to be Fourier series in space. They where then able to derive the equation

$$
\begin{aligned}
\gamma= & \alpha \omega+2 \alpha \omega \sum_{n=-\infty}^{\infty} \frac{\Gamma^{2} \sin ^{2}((\Gamma L-n \pi) / 2)}{L^{2}(\Gamma-n \pi / L)^{2}(\Gamma+n \pi / L)^{2}} \\
& \times\left(\frac{\sin ^{2} v_{0}}{\left(\omega^{2}-c_{-}^{2} n^{2} \pi^{2} / L^{2}\right)^{2}+\alpha^{2} \omega^{2}}\right. \\
& \left.+\frac{\cos ^{2} v_{0}}{\left(\omega^{2}-c_{+}^{2} n^{2} \pi^{2} / L^{2}\right)^{2}+\alpha^{2} \omega^{2}}\right),
\end{aligned}
$$

approximating the current-voltage $(\gamma-\omega)$ characteristic of the two coupled junctions. The voltage of each junction $(\omega)$ is assumed to be identical, $v_{0}=\phi_{0}^{2}-\phi_{0}^{1}$ expresses the phase difference between the two junctions, and $c_{ \pm}^{2}=1 /(1 \pm S)$ are the characteristic velocities squared.

Following Ref. 13 for the case of a single junction, Eq. (8) may be solved numerically to yield the $\omega-\Gamma$ characteristic at constant bias current, which is often measured in experiments. Unlike the case of a single junction, an undetermined phase difference $v_{0}$ enters the calculations in the case of two coupled junctions. Since fluxons in different junctions repel each other, ${ }^{21}$ the intuitively most stable configuration of a fluxon lattice is when the fluxon-fluxon distance is largest, i.e., the antiphase vortex configuration corresponding to $v_{0}=\pi / 2$. Nevertheless, the in-phase vortex configuration, corresponding to $v_{0}=0$, has in zero magnetic field been shown to be dynamically stable, ${ }^{22}$ and it is also the most interesting configuration for applications. In Fig. 2, we have plotted the $\omega(\Gamma)$ curve for both cases, by solving Eq. (8) iteratively. We start at $\Gamma=50$ and then solve Eq. (8) numerically to obtain $\omega$, using $\omega=\gamma / \alpha$ as an initial value [as $\omega(\Gamma) \rightarrow \gamma / \alpha$ for $\Gamma \rightarrow \infty]$. Then $\Gamma$ is stepped down by a small amount and the previously found value of $\omega$ is used as an initial value for the numerical solution of Eq. (8) with the new and smaller value of $\Gamma$. This is repeated until we reach $\Gamma=0$. In the plots, we have marked $\Gamma=n \pi / L, n=0,1, \ldots$ by vertical lines, corresponding to the different Fiske steps. For $\operatorname{large} \Gamma$, we see a periodic behavior with a period of $2 \pi / L$. At low values of $\Gamma$, we see some complex behavior, especially for $v_{0}=0$. We do not see oscillations with period $\pi / L$ in these plots. We suspect this is because of the small length. It is important to note, that we see the $2 \pi / L$ oscillations for both the in-phase solution and the antiphase solution.

The system of nonlinear Eqs. (1)-(5) has been solved numerically using second order finite differences for the spatial derivatives and a fifth order Runge-Kutta method for the time evolution. Starting with $\phi^{i}=0(i=1, \ldots, N)$ and $\Gamma=0$, the equations have been solved numerically from $t=0$ to $t_{m}$. $\Gamma$ is then increased by a small amount, keeping all other parameters fixed. With the previous solution as initial conditions, a new solution is generated, again from $t=0$ to $t_{m}$. This is repeated until a maximum value of $\Gamma$ is reached. Taking care that $t_{m}$ is large enough for the system to reach a possible steady state at each value of $\Gamma$, the voltage vs applied magnetic field characteristic of the system is obtained. Since the individual voltages, 

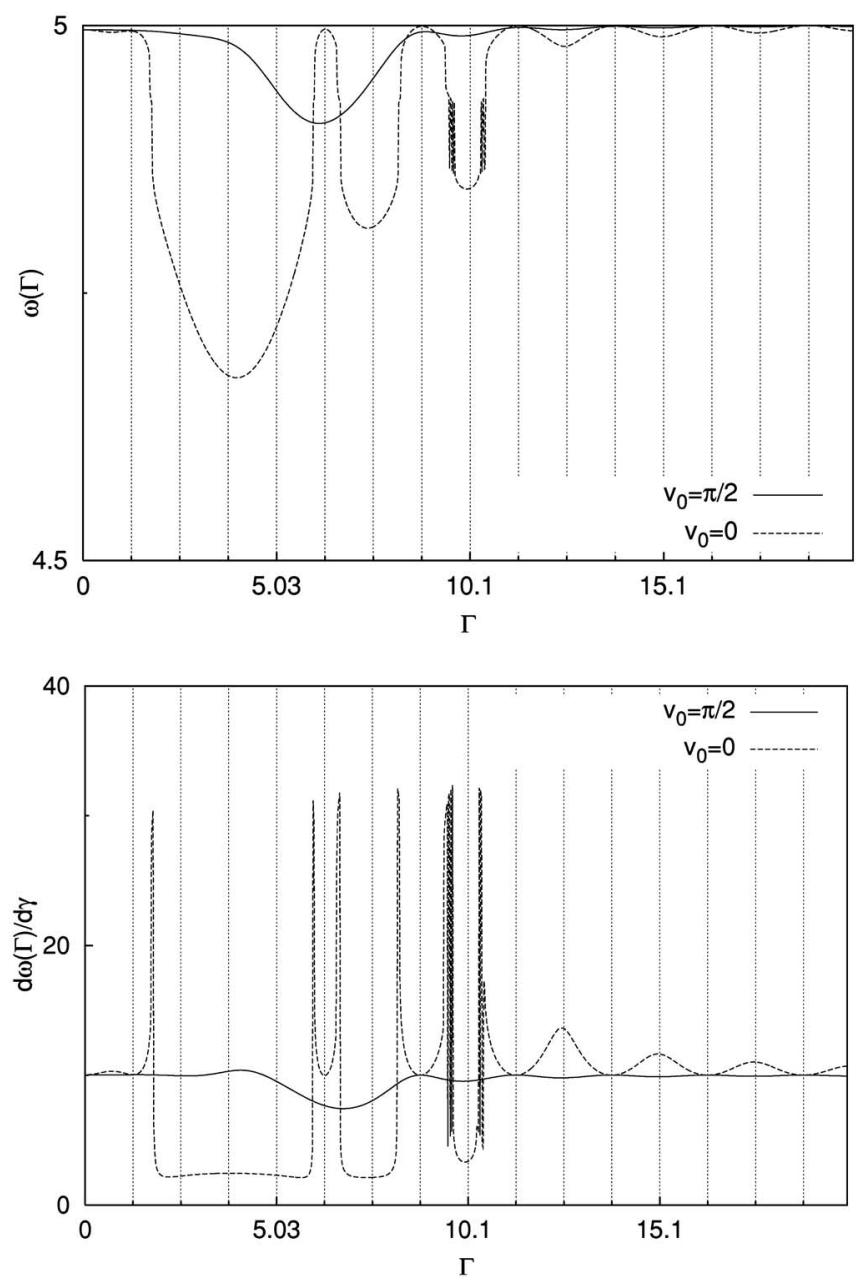

FIG. 2. Curves obtained by solving Eq. (8) numerically with $v_{0}=0$ and $v_{0}=\pi / 2$ to obtain the voltage, $\omega(\Gamma)$ (top), and the dynamic resistance, $d \omega(\Gamma) / d \gamma$ (bottom). Vertical lines at $\Gamma=n \pi / L$, $n=0,1, \ldots$ Parameters used: $L=2.5, \alpha=0.1, \gamma=0.5, S=-0.4$.

$$
\omega^{i}=\lim _{t \rightarrow \infty} \frac{1}{L\left(t-t_{0}\right)} \int_{t_{0}}^{t} \int_{0}^{L} \phi_{t}^{i} d x d t
$$

cannot be assumed to be identical for all junctions, the average voltage,

$$
V \equiv \frac{1}{N} \sum_{i=1}^{N} \omega^{i}
$$

has been computed. To get a feeling of the total number of fluxons in the system, the phase difference,

$$
\Delta \phi \equiv \sum_{i=1}^{N}\left(\phi^{i}(L)-\phi^{i}(0)\right),
$$

is also calculated. This expression is approximately $2 \pi$ times the number of fluxons in the system.

Figure 3 presents the results of the above method used on two stacked junctions. To investigate in detail what happens in the characteristics, we plot $\cos \left(\phi^{i}\right)$ for each junction at selected values of $\Gamma$. Marked on these figures are the points $\cos \left(\phi^{i}\right)=-1$, which approximates the position of a fluxon. Thus, the fluxon configuration can directly be seen at different places in the $V$ - $\Gamma$ characteristic. For very low magnetic field (a), there are no fluxons in the junctions. At some small field (b), about $\Gamma=2$, fluxons starts to enter the junctions and a large increase in the average voltage is observed. The fluxons are in an antiphase configuration. Increasing the field a little more to point (c), the voltage drops a little and the two fluxons in the junctions have rearranged themselves from the antiphase configuration to the in-phase configuration. Increasing the field to (d), more fluxons enter the junctions, until we observe another drop in the voltage at point (e), where the fluxons have jumped from the in-phase configuration to the antiphase configuration. As the field is increased to point (e), the voltage remains essentially constant but more and more fluxons enter the system in an antiphase configuration. At point $(\mathrm{g})$, the voltage jumps up to about $\gamma / \alpha$ and from here on, the $2 \pi / L$ oscillations appear and the fluxon always seems to be in an antiphase configuration (h). Note that the $2 \pi / L$ oscillations appear when the phases seem approximately linear as a function of $x$. Judging from our calculations, this happens when $\Delta \phi /(2 \pi L N) \approx 1$, i.e., when the length per fluxon per junction is about unity.

The phase difference, $\Delta \phi$, in Fig. 3 shows an almost linear dependence on $\Gamma$, though small jumps on the curve can be observed. This fits well with Eqs. (6) and (7), which give $\Delta \phi \approx N L \Gamma$, neglecting $\delta \phi^{i}$. Since $\Delta \phi$ increases almost linearly, we conclude that the fluxon entry happens almost continuously.

In Fig. 4, we present the $V$ - $\Gamma$ characteristic for a system with identical parameters as the one in Fig. 3, but with a smaller bias current, $\gamma=0.2$. For large magnetic field, we see the $2 \pi / L$ oscillations, which start at a value of $\Gamma$ a little smaller than it was for the case with $\gamma=0.5$. Again we see the in-phase configuration of the vortices, but for a different region of $\Gamma$, though still for $\Gamma$ below the $2 \pi / L$-oscillation regime.

In Fig. 5, the length of the system has been increased to $L=10$. This gives room for more fluxons in each junction. For large magnetic field, we see again the $2 \pi / L$-oscillations. In contrast to the short systems in Figs. 3 and 4, we only observe the in-phase mode for a high value of the bias current, and even here the range of magnetic field where the in-phase mode is observed is severely shortened as compared to the $L=2.5$ system. The in-phase mode starts at $\Gamma \approx 3$ and ends at $\Gamma \approx 3.7$. For slightly higher values of $\Gamma$, the in-phase mode starts to become erratic. We do observe square lattice for some $\Gamma$ values in this range, but a slight increase in $\Gamma$ may change the structure of the lattice. This is also evident from the dynamic resistance plot, where the region labeled "Partial in-phase" shows large peaks signaling instability.

\section{MORE JUNCTIONS}

In order to use stacked Josephson junctions for oscillators in applications, the power of the emitted radiation must be high. One way to obtain this is by increasing the number of 

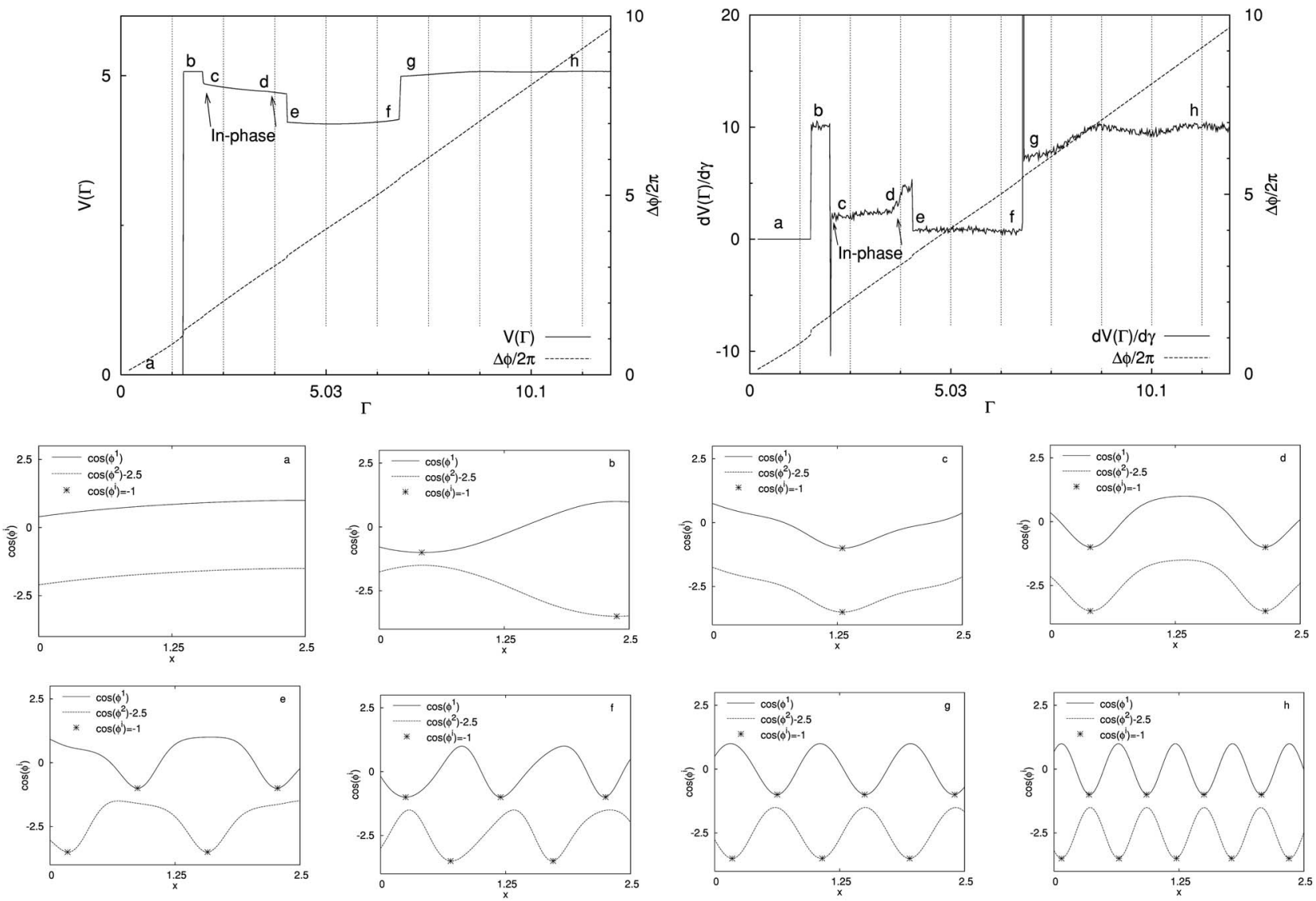

FIG. 3. Two junctions, high bias current. Average voltage, $V(\Gamma)$ (top left), and dynamic resistance, $d V(\Gamma) / d \gamma($ top right), obtained by a numerical simulation of Eqs. (1)-(5). Lower plots, (a)-(h), show $\cos \left(\phi^{i}\right)$ at selected values of $\Gamma$. Vertical lines at $\Gamma=n \pi / L, n=0,1, \ldots$. Parameters used: $L=2.5, \alpha=0.1, \gamma=0.5, S=-0.4$. The in-phase fluxon configuration is observed in the range of magnetic field between (c) and (d).

junctions in the stack and operated the stack in the in-phase mode. As it was shown in Sec. III, we seem to get the square lattice most easily for a short system and a high bias current. Shown in Fig. 6 are calculations on systems with five, seven, and ten junctions. The overall picture is the same as two junctions, but some added complexity arising from the extra degrees of freedom is found. Fluxons enter around $\Gamma=2$ and at high values of $\Gamma$ the $2 \pi / L$ oscillations are found, with the
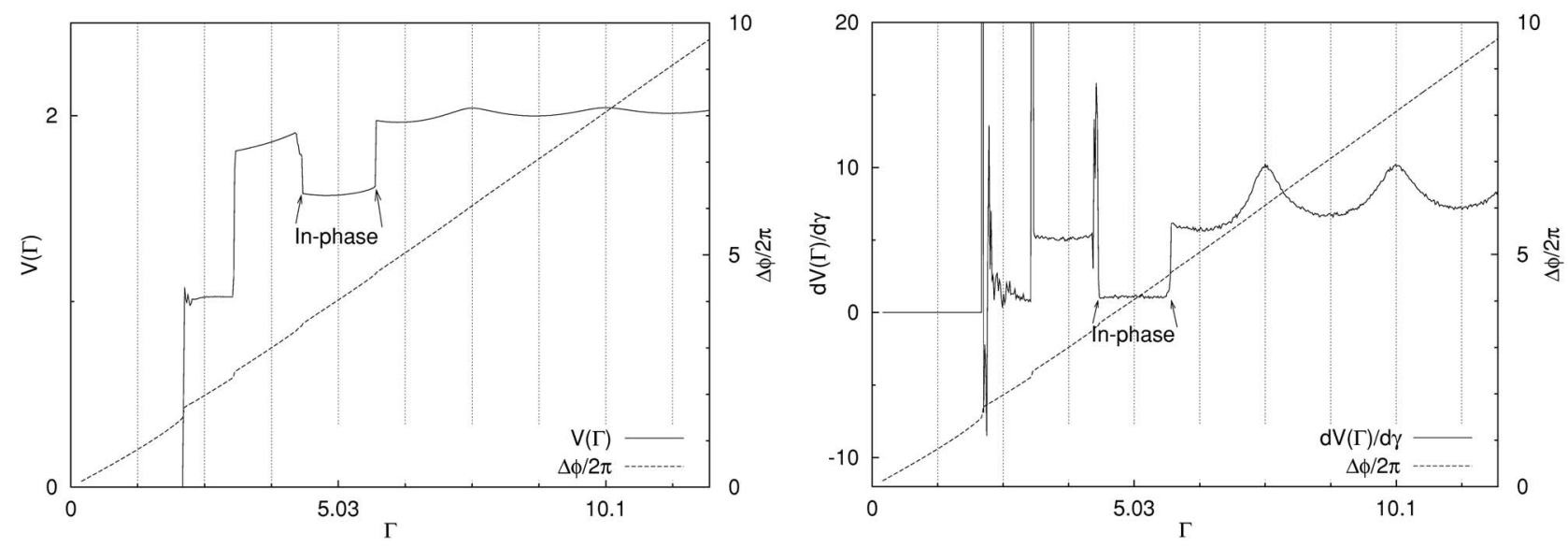

FIG. 4. Two junctions, small bias current. Average voltage, $V(\Gamma)$ (top), and dynamic resistance, $d V(\Gamma) / d \gamma($ bottom), obtained by a numerical simulation of Eqs. (1)-(5). Vertical lines at $\Gamma=n \pi / L, n=0,1, \ldots$. Parameters used: $L=2.5, \alpha=0.1, \gamma=0.2, S=-0.4$. In-phase vortex configuration is observed, but at higher values of $\Gamma$ than for the higher bias current case shown in Fig. 3. 

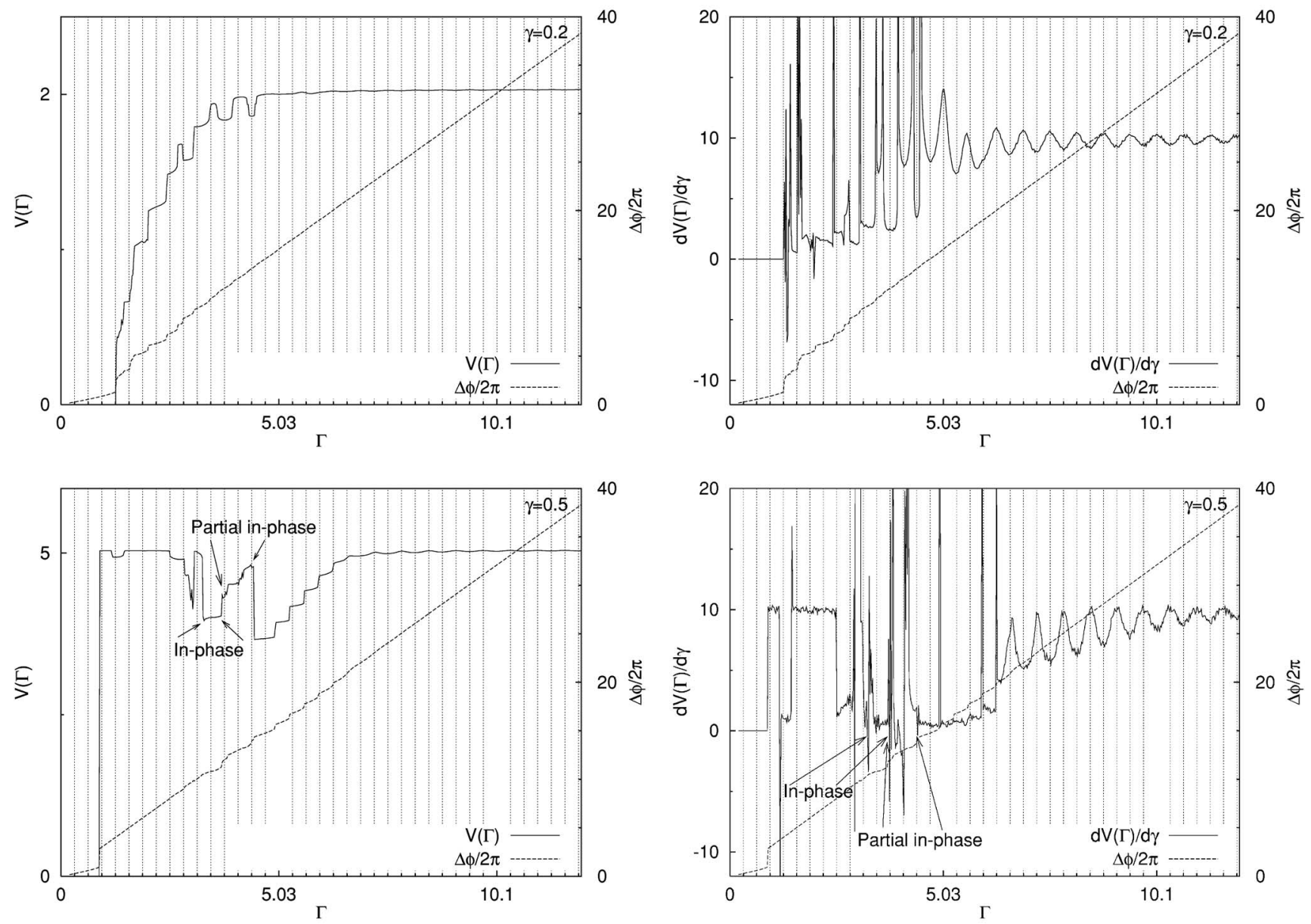

FIG. 5. Two junctions, $L=10$. Average voltage, $V(\Gamma)$ (left), and dynamic resistance, $d V(\Gamma) / d \gamma$ (right), obtained by a numerical simulation of Eqs. (1)-(5). Vertical lines at $\Gamma=n \pi / L, n=0,1, \ldots$. Parameters used: $L=10, \alpha=0.1, \gamma=0.2$ (top), and 0.5 (bottom), $S=-0.4$. Only for the high value of the bias current is the in-phase configuration observed.

fluxons always arranged in a triangular lattice. Between these two values, complicated behavior is observed. Here the vortex lattice is not very rigid due to the low fluxon density and it may be distorted considerably by the perturbations arising from the edges. In this "intermediate" region, the lattice is quite erratic, but usually arranged in a triangular fashion due to the repulsiveness of the fluxons. But typically, a small region of $\Gamma$ values shows the interesting square lattice. Unfortunately, this region seems to become smaller when the number of junctions increase, at least for the finite number of calculations we have done. Also, looking at the plots in Fig. 6 , we see that for the cases of seven and ten junctions, the fluxon density in the range of the in-phase configuration is seemingly below one fluxon per junction, while for the cases of 2 (Fig. 3) and five junctions, it may be higher. The plots of the individual phases do, however, show one fluxon in each junction arranged in the in-phase configuration for seven (bottom of Fig. 7) and ten (not shown) junctions. Unlike the case of $N=2$, where the fluxons are exactly identical in the in-phase configuration, the fluxons for $N>2$ in the in-phase configuration show some dissimilarities and they may be positioned very slightly out of phase, see bottom plot of Fig. 7. Also, since the fluxon density per junction where the in-phase configuration is observed decreases with an increase in the number of junctions, we conclude that the system is having trouble phase locking a large number of fluxons.

In the plots for $N=7$ and $N=10$ in Fig. 6, the in-phase configuration is observed close to the first fluxon entry. Since we start the numerical code with $\phi^{i}=0$ for $i=1, \ldots, N$, one might suspect that the in-phase configuration is, in these cases, only obtained due to the symmetry of our initial conditions. Shown in Fig. 7 is $\cos \left(\phi^{i}\right)$ for $\Gamma=1.6$ and $\Gamma=1.8$ taken from the calculations with $N=7$ in Fig. 6 . We observe, that before the in-phase configuration is obtained at $\Gamma=1.8$, there is a transition from zero fluxons to a state, at $\Gamma=1.6$, where only some of the junctions contains fluxons, thus the "in-phase symmetry" of the initial conditions has been broken.

Calculations on longer junctions and different values of the bias current have been made but not shown. The larger length allows for more "freedom" in the region of low fluxon density, giving rise to even more complicated characteristics. For high values of the magnetic field, the system always 

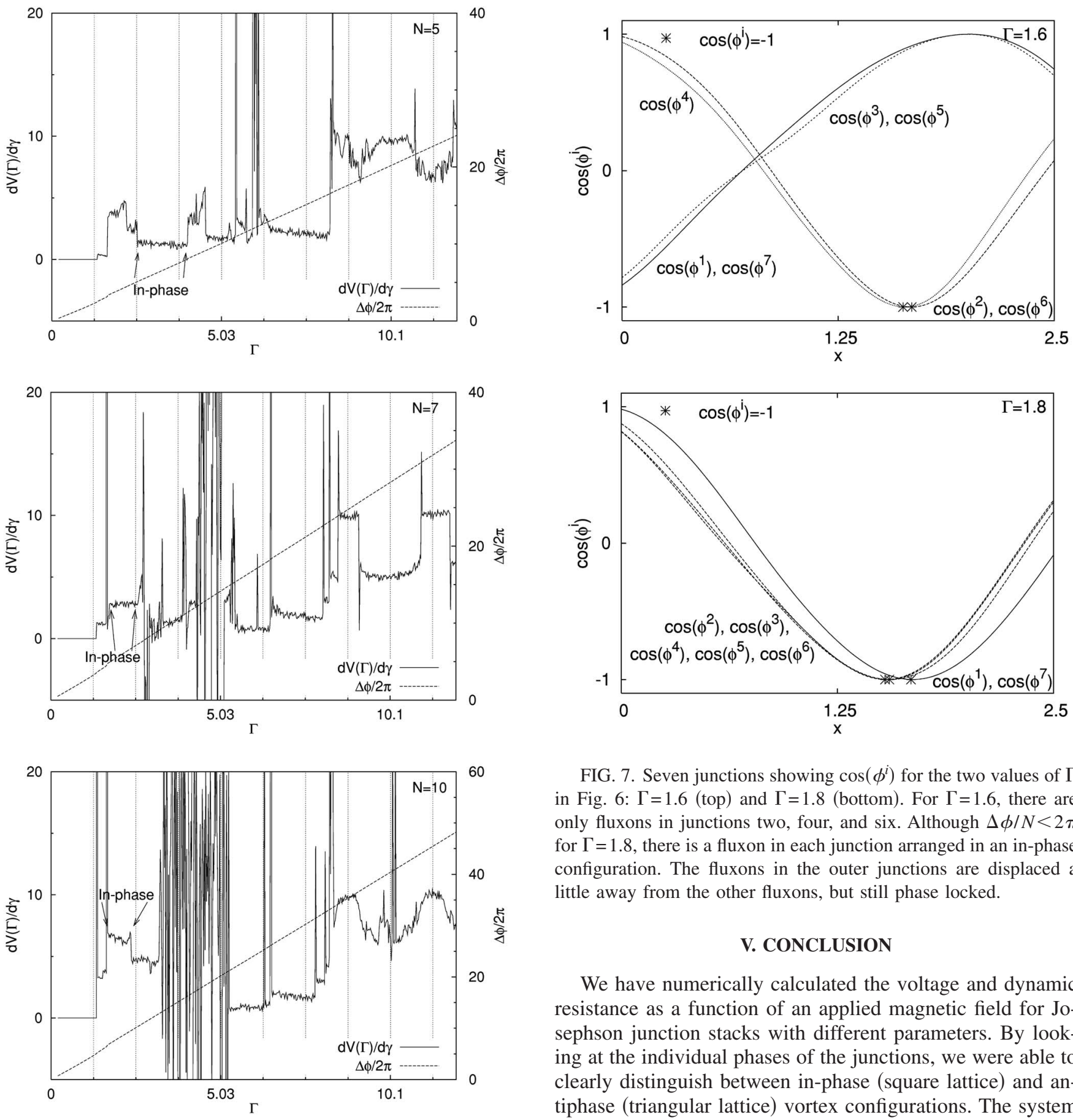

FIG. 7. Seven junctions showing $\cos \left(\phi^{i}\right)$ for the two values of $\Gamma$ in Fig. 6: $\Gamma=1.6$ (top) and $\Gamma=1.8$ (bottom). For $\Gamma=1.6$, there are only fluxons in junctions two, four, and six. Although $\Delta \phi / N<2 \pi$ for $\Gamma=1.8$, there is a fluxon in each junction arranged in an in-phase configuration. The fluxons in the outer junctions are displaced a little away from the other fluxons, but still phase locked.

\section{CONCLUSION}

We have numerically calculated the voltage and dynamic resistance as a function of an applied magnetic field for Josephson junction stacks with different parameters. By looking at the individual phases of the junctions, we were able to clearly distinguish between in-phase (square lattice) and antiphase (triangular lattice) vortex configurations. The system was mostly found to have a triangular vortex lattice, or a similar noncoherent vortex configuration. The square lattice was observed in some systems. Small length, high bias current, and a low number of junctions seemed to enhance the conditions for a square lattice, which was observed only for some values of the magnetic field. The structure of the vortex lattice was not found to be connected to oscillations in the voltage vs magnetic field curves.

\section{ACKNOWLEDGMENTS}

seem to exhibit $2 \pi / L$ oscillations with the fluxons always arranged in a triangular lattice, and the longer junctions seem to be less likely to exhibit in-phase configuration, like in the case of two junctions considered in Sec. III.

We acknowledge the support from the STVF framework programme "New Superconductors: Mechanisms, processes, and products." 
*Electronic address: Soeren@Madsen2TheMax.dk

†Electronic address: nfp@oersted.dtu.dk

${ }^{1}$ R. Kleiner and P. Muller, Phys. Rev. B 49, 1327 (1994).

${ }^{2}$ A. A. Yurgens, Supercond. Sci. Technol. 13, R85 (2000).

${ }^{3}$ S. Sakai, P. Bodin, and N. F. Pedersen, J. Appl. Phys. 73, 2411 (1993).

${ }^{4}$ T. Koyama and M. Tachiki, Phys. Rev. B 54, 16183 (1996).

${ }^{5}$ S. Madsen and N. F. Pedersen, Phys. Rev. B 69, 064507 (2004).

${ }^{6}$ M. Machida, Phys. Rev. Lett. 90, 037001 (2003).

${ }^{7}$ S. Ooi, T. Mochiku, and K. Hirata, Phys. Rev. Lett. 89, 247002 (2002).

${ }^{8}$ I. Kakeya, M. Iwase, T. Yamamoto, and K. Kadowaki, Proceedings of the Plasma 2004 Conference, Tsukuba, Japan, II-90, 2004 (unpublished); I. Kakeya and K. Kadowaki (private communication).

${ }^{9}$ T. Hatano, H. Wang, S. Kim, S. Urayama, S. Kawakami, S. J. Kim, M. Nagao, K. Inomata, Y. Takano, T. Yamashita, and M. Tachiki, IEEE Trans. Appl. Supercond. 15(2), 912 (2005).

${ }^{10}$ S. Madsen, G. Filatrella, and N. F. Pedersen, Eur. Phys. J. B 40, 209 (2004).

${ }^{11}$ N. F. Pedersen and S. Madsen, IEEE Trans. Appl. Supercond. 15(2), 948 (2005).

${ }^{12}$ N. F. Pedersen and S. Madsen, Proceedings of the Plasma 2004 Conference, Tsukuba, Japan, II-48, 2004 (unpublished).
${ }^{13}$ A. V. Ustinov and N. F. Pedersen, Phys. Rev. B 72, 052502 (2005).

${ }^{14}$ I. O. Kulik, Zh. Eksp. Teor. Fiz. Pis'ma Red. 2, 134 (1965) [JETP Lett. 2, 84 (1965)]; M. Cirillo, N. Grønbech-Jensen, M. R. Samuelsen, M. Salerno, and G. V. Rinati, Phys. Rev. B 58, 12377 (1998).

${ }^{15}$ S. Kim, S. Urayama, H. B. Wang, M. Nagao, Y. Takano, and T. Hatano, Proceedings of the Plasma 2004 Conference, Tsukuba, Japan, II-149, 2004 (unpublished).

${ }^{16}$ I. Kakeya, M. Iwase, T. Yamazaki, T. Yamamoto, and K. Kadowaki, 2005 APS March Meeting Session B12, 2005 (unpublished).

${ }^{17}$ Yu. I. Latyshev, A. E. Koshelev, and L. N. Bulaevskii, Phys. Rev. B 68, 134504 (2003).

${ }^{18}$ A. E. Koshelev and I. Aranson, Phys. Rev. B 64, 174508 (2001).

${ }^{19}$ N. F. Pedersen, in Solitons, edited by A. A. Maradudin and V. H. Agranowich (North-Holland, Amsterdam, 1986), p. 469.

${ }^{20}$ N. Grønbech-Jensen, J. A. Blackburn, and M. R. Samuelsen, Phys. Rev. B 53, 12364 (1996).

${ }^{21}$ N. Grønbech-Jensen, M. R. Samuelsen, P. S. Lomdahl, and J. A. Blackburn, Phys. Rev. B 42, 3976 (1990).

${ }^{22}$ N. Grønbech-Jensen, D. Cai, and M. R. Samuelsen, Phys. Rev. B 48, R16160 (1993). 